\title{
Thermal niches of specialized gut symbionts: the case of social bees
}

Tobin J. Hammer*, Eli Le, Nancy A. Moran

Department of Integrative Biology, University of Texas at Austin, Austin, TX 78703

*Corresponding author: tobin.hammer@utexas.edu

Keywords: microbiome, Apis, Bombus, heat stress, thermoregulation, Snodgrassella 


\section{Abstract}

Responses to climate change are particularly complicated in species that engage in

4 symbioses, as the niche of one partner may be modified by that of the other. We explored

5 thermal traits in gut symbionts of honeybees and bumblebees, which are vulnerable to rising

6 temperatures. In vitro assays of symbiont strains isolated from 16 host species revealed variation

7 in thermal niches. Strains from bumblebees tended to be less heat-tolerant than those from

8 honeybees, possibly due to bumblebees maintaining cooler nests or inhabiting cooler climates.

9 Overall however, bee symbionts grew at temperatures up to $44{ }^{\circ} \mathrm{C}$ and withstood temperatures up

10 to $52{ }^{\circ} \mathrm{C}$, at or above the upper thermal limits of their hosts. While heat-tolerant, most strains of

11 the symbiont Snodgrassella grew relatively slowly below $35^{\circ} \mathrm{C}$, perhaps because of adaptation

12 to the elevated body temperatures that bees maintain through thermoregulation. In a gnotobiotic

13 bumblebee experiment, Snodgrassella was unable to consistently colonize bees reared below 35

$14{ }^{\circ} \mathrm{C}$ under conditions that limit thermoregulation. Thus, host thermoregulatory behavior appears

15 important in creating a warm microenvironment for symbiont establishment. Bee-microbiome-

16 temperature interactions could affect host health and pollination services, and inform research on

17 the thermal biology of other specialized gut symbionts, such as those of humans.

\section{Introduction}

Earth's climate is rapidly warming, and there is an urgent need to understand how

22 organisms will respond [1,2]. One factor complicating such predictions is the role of interspecific

23 interactions $[3,4]$, and, in particular, symbiosis [5,6]. Many organisms closely associate with one 
24 or more distantly related partners that have highly distinct physiologies, as in the case of animal

25 or plant hosts and their microbiomes. Hosts and microbes are likely to have different responses

26 to temperature; yet, if they are mutually dependent, the combined niche is restricted to that of the

27 more sensitive partner. Furthermore, thermal niches can themselves evolve in response to

28 symbiotic lifestyles. For example, obligate endosymbionts that undergo strong population

29 bottlenecks during transmission may evolve unstable, easily denatured proteins as a consequence

30 of mutation accumulation, leading to heat sensitivity [7-9]. Both of these factors may constrain

31 the combined thermal niche of strongly symbiont-dependent organisms $[6,10,11]$. There is

32 evidence for symbiont-imposed constraints on host thermotolerance in a variety of invertebrates

33 such as aphids, ants, stinkbugs, corals, and sponges [12-16]. However, the wider prevalence of

34 this phenomenon is unclear, and, in general, we do not know how microbiomes will influence

35 host responses to climate warming.

The eusocial corbiculate bees (hereafter "social bees") are a particularly important group

37 in which to study symbiont thermal niches and their effects on hosts. This clade, comprising

38 honey bees (Apis), bumblebees (Bombus), and stingless bees (Meliponini), is host to anciently

39 associated, host-specialized, and beneficial gut microbiomes [17,18]. Social bees are also key

40 pollinators in both agricultural and natural ecosystems, but many are declining [19,20]. For

41 bumblebees in particular, rising temperatures have been identified as a driver of range shifts and

42 population declines in some species $[21,22]$. If gut symbionts are sensitive to heat stress, the

43 microbiome could be one route through which climate change impacts bee health. Furthermore,

44 because social bees as a whole exhibit extensive strain-level diversity in their microbiomes (e.g.,

$45[17,23])$, strain variability in thermotolerance could partially underlie corresponding variability

46 among hosts, as was recently shown for endosymbionts of aphids [24]. 
Social bees present a uniquely complex thermal environment for their microbiome, making it challenging to predict their symbionts' thermal traits. They are not strictly

49 poikilothermic; rather, they facultatively regulate the temperature of both their bodies (and

50 individual body parts) as well as their shared nests [25-27]. These microenvironments are

51 partially buffered from external fluctuations in temperature, but to a degree that is highly

52 dynamic among individuals, over time and space, and across the bee phylogeny. Even within a

53 single nest, the microbiome is distributed across individuals that vary in behaviors such as

54 foraging or brood incubation, which involve changes in host body temperature [28-30].

55 Furthermore, social bee species regulate their nest temperatures to different set-points and

56 exhibit different overwintering strategies [27,31,32]. For example, the microbiomes of

57 temperate-zone bumblebees must overwinter within diapausing queens, while the microbiome of

58 Apis mellifera is transmitted by a cluster of active, heat-generating workers [33,34].

As in gut symbionts generally, symbionts of social bees experience a brief ex vivo phase

60 during transmission, potentially imposing selection on thermal traits. The gut microbiome is

61 transmitted via a fecal-oral route, usually between nestmates within a hive [35,36], but horizontal

62 transmission between bee species has also been inferred [17,34]. Although the symbionts cannot

63 grow under ambient oxygen levels outside the bee gut [18], the ability to tolerate thermal stress

64 while on flowers or other external habitats could influence horizontal transmission rates and thus

65 patterns of biogeography and host specificity. All of these factors add up to a complex selective

66 landscape - even within a single host species - involving different castes, seasons, and ex vivo

67 phases.

Very little is currently known about the thermal biology of social bee microbiomes.

69 Recent work on Bombus impatiens has shown that, once established in the gut, core symbionts 
are relatively robust to temperatures from $21-37^{\circ} \mathrm{C}[37]$. However, honeybees and bumblebees emerge largely symbiont-free as adults and must acquire their symbionts from nestmates

$72[35,36,38]$. It is not known if the colonization process, a crucial phase for both hosts and

73 symbionts, is more temperature-sensitive than maintenance of an established symbiosis.

74 Furthermore, there has been no comparative work investigating how symbiont thermal traits

75 have evolved across social bees. Social bee taxa maintain different nest temperatures and use different strategies to overwinter and to establish new colonies. They also occupy a climatically

77 diverse range of environments, from arctic and alpine habitats to tropical forests [39]. This

78 variability may impose divergent selection on symbiont thermal traits, with potential feedbacks on the thermal tolerance of the hosts themselves.

We used common garden experiments (in vitro) to characterize symbiont thermal niches

81 with a culture collection of Snodgrassella and Gilliamella, two bacterial species that are

82 ubiquitous across honeybees and bumblebees [17]. Symbionts of these two bee lineages belong

83 to deeply divergent clades and appear restricted to their native host $[17,40]$. We measured the

84 thermal limits to growth from $12-48{ }^{\circ} \mathrm{C}$, the ability to tolerate a brief heat exposure up to $52{ }^{\circ} \mathrm{C}$,

85 and growth rates at $28{ }^{\circ} \mathrm{C}$ versus $35^{\circ} \mathrm{C} .28^{\circ} \mathrm{C}$ is within the range of brood nest temperatures

86 reported for some bumblebee species [41,42] (though not all [43]), while honeybee brood nest

87 temperatures are typically $\sim 33-36{ }^{\circ} \mathrm{C}[31,32,44]$, at least for Apis mellifera and A. cerana.

We hypothesized that for all three metrics, honeybee-associated strains would exhibit

89 higher thermotolerance than bumblebee-associated strains, as a result of adaptation to a generally

90 warmer host (nests and bodies) and external environment. We also examined whether the

91 thermal environment impacts Snodgrassella establishment in the bumblebee Bombus impatiens.

92 This experiment examined whether the colonization process is particularly vulnerable to thermal 
93 stress; our initial hypothesis was that an abnormally high ambient temperature for $B$. impatiens

$94\left(35^{\circ} \mathrm{C}\right)$ would impair symbiont acquisition. Our findings provide a foundation for future work on

95 the thermal ecology of bee gut microbiomes and raise new questions about the role of host

96 thermoregulatory behavior in mediating symbiosis.

97

98 Materials \& Methods

99

100

\section{Culture collection}

101 Strains of Snodgrassella alvi and Gilliamella spp. used in this study were collected as

102 described in refs. [40,45,46]. To our knowledge, no strains had undergone significant passaging

103 in the lab since the original isolates were obtained, with the exception of Snodgrassella alvi

104 strain wkB2. Bumblebee host species were categorized into high-elevation or low-elevation

105 species for Figs. S1, S2 following range descriptions in ref. [47].

Thermal limits assay

From glycerol stocks, strains of Snodgrassella or Gilliamella were cultured on Columbia

109 blood agar plates under $5 \% \mathrm{CO}_{2}$ and $35^{\circ} \mathrm{C}$ for $2 \mathrm{~d}$. These were then restreaked, and the overnight

110 cultures were resuspended in Insectagro medium (Corning) and adjusted to an $\mathrm{OD}_{600}$ of 0.5 . We

111 further diluted these cell suspensions $1 / 20$ and spotted $10 \mu 1$ in triplicate onto the surface of fresh

112 Columbia plates. Negative controls (10 $\mu 1$ Insectagro spotted onto plates) were included to

113 ensure there was no background contamination. Given that the same OD can correspond to

114 different densities of viable cells, we also quantified the corresponding colony-forming unit

115 (CFU) count of the inoculum for each strain. Plates were incubated at a range of temperatures 
116 under $5 \% \mathrm{CO}_{2}$, from $12-48{ }^{\circ} \mathrm{C}$ in increments of $4{ }^{\circ} \mathrm{C}$. After $48 \mathrm{~h}$, we scored these plates for

117 whether visible biomass was present or absent. If a strain could not grow at or below $28^{\circ} \mathrm{C}$, it

118 was not tested at colder temperatures. For example, strain wkB339 could not grow at $28{ }^{\circ} \mathrm{C}$ so it

119 was not further tested at $24{ }^{\circ} \mathrm{C}$ or below.

120 We used logistic regression (implemented in R [48] as a generalized linear model with

121 binomial error and logit link function), to test whether host genus (Apis vs. Bombus) predicted

122 the ability of symbionts to grow at $44^{\circ} \mathrm{C}$, the upper thermal limit across strains in this assay. To

123 account for the potential influence of starting inoculum size on the probability of growth, we

124 used the same approach, but with log-transformed CFU counts for each strain as a predictor.

Heat stress assay

Snodgrassella cultures were initially prepared as above. We then resuspended overnight

128 cultures in Insectagro, adjusted them to an $\mathrm{OD}_{600}$ of 0.5 , and further diluted $1 / 20$ in $200 \mu \mathrm{in}$

129 PCR plates. Cells were subjected to a $1 \mathrm{~h}$ heat stress treatment using a thermocycler with a

130 temperature gradient from $35.4^{\circ} \mathrm{C}$ to $51.6^{\circ} \mathrm{C}$ (3 technical replicates per strain per temperature).

131 They were then transferred to 96-well cell culture plates (Corning) in a microplate reader (Tecan)

132 with $5 \% \mathrm{CO}_{2}$ and $35^{\circ} \mathrm{C}$. Growth was monitored by $\mathrm{OD}_{600}$ readings taken every $3 \mathrm{~h}$ for $66 \mathrm{~h}$. We

133 included blank wells (Insectagro only) as negative controls and subtracted their OD 600 values

134 from those of the cultures.

Thermal performance assay

137 Snodgrassella cultures were prepared as for the heat stress assay, except that they were

138 transferred directly into 96-well cell culture plates in the microplate reader (without the 
139 thermocycler step) and incubated at either $28^{\circ} \mathrm{C}$ or $35^{\circ} \mathrm{C}$ for $72 \mathrm{~h}$. We fit logistic curves to the

140 data using the growthcurver package [49] and used a two-way ANOVA to test for effects of

141 incubation temperature and host genus on the intrinsic growth rate $(r)$. Linear regression was

142 used to test whether starting inoculum size (log-transformed CFU counts) predicted growth rate.

144 Colonization experiment

145 To obtain gnotobiotic bumblebees, we collected clumps of pupal cocoons from four 146 separate commercial colonies of Bombus impatiens (Koppert USA). We then surface-sterilized

147 the clumps in diluted bleach $(0.2 \% \mathrm{NaOCl})$ for $90 \mathrm{~s}$ as described previously [50,51] to minimize

148 contamination of the emerging workers. We maintained the sterilized cocoon clumps in sterile

149 conditions in a growth chamber at $35^{\circ} \mathrm{C}$ and monitored them daily for adult emergence. Newly

150 emerging adults were transferred to sterile vials and randomly assigned to Snodgrassella or

151 buffer-only treatments. The former were fed with $10 \mu 1$ of filter-sterilized sugar syrup (50\% v/v)

152 containing $\sim 10^{6}$ cells of Snodgrassella alvi strain wkB12, following [40]. We prepared new

153 Snodgrassella inocula daily from overnight cultures; these were not continuously propagated but

154 rather independently obtained from the same frozen stock of wkB12. Negative-control bees were

155 fed an identical solution but without cells. All bees were monitored to ensure that they consumed

156 the entire $10 \mu 1$ of inoculum.

157 We then transferred bees to sterilized 16 oz plastic containers (Dart Container Corp.) in

158 groups of 2-3 as microcolonies [52]. Bees within a microcolony were assigned to the same

159 treatment and were obtained from the same source colony. Each container was provided with 10

$160 \mathrm{ml}$ of sterile $50 \%$ sugar syrup and $500 \mathrm{mg}$ of sterile pollen dough (ground gamma-irradiated

161 honeybee pollen mixed with sterile 50\% sugar syrup). Microcolonies were reared in incubators at 
$16229^{\circ} \mathrm{C}$, representing a typical bumblebee rearing temperature $[37,52,53]$, or $35^{\circ} \mathrm{C}$, a temperature 163 typical of Apis mellifera hives [32]. The pollen lump was replaced on the third day of rearing.

After $5 \mathrm{~d}$, bees were briefly anaesthetized in ice and used for gut dissections. The gut

165 (including hindgut and midgut) was removed from each bee, homogenized with a sterile plastic

166 pestle, and resuspended in $1 \mathrm{ml}$ of Insectagro. This homogenate was serially diluted and plated

167 on Columbia blood agar. We counted CFUs after $2 \mathrm{~d}$ of incubation at $35{ }^{\circ} \mathrm{C}$ in $5 \% \mathrm{CO}_{2}$. In

168 performing CFU counts of the focal Snodgrassella strain, we noticed the occasional presence (in

$16915 \%$ of bees overall) of an unidentified bacterium. This contaminant had distinct colony

170 morphology and exhibited slow growth and hemolysis. There was no significant association

171 between the inoculum or the rearing temperature with the presence of the contaminant (logistic

172 regression; inoculum $p=0.19$, temperature $p=0.066$ ). This may have been a core bee gut

173 symbiont not completely removed from the cocoon surface by the sterilization treatment.

174 We used a linear mixed-effects model with the nlme package [54], treating colony source

175 as a random effect, to test whether rearing temperature was associated with log-transformed

176 Snodgrassella CFU counts. We also used logistic regression (as described above) to test whether

177 bee survival was predicted by the inoculum treatment or by rearing temperature.

179 Results

We first used an in vitro assay to measure the temperature limits of growth of a collection

182 of Snodgrassella and Gilliamella strains isolated from honeybees and bumblebees. While all

183 strains could grow at $40{ }^{\circ} \mathrm{C}$ (Fig. S1) and none at $48^{\circ} \mathrm{C}$, they varied in their ability to grow at 44 
$184{ }^{\circ} \mathrm{C}$ (Fig. 1). Bumblebee strains were significantly less likely to be able to grow at this

185

186 temperature than honeybee strains (logistic regression, $p=0.015$ ).

There was also substantial strain-level variation in lower thermal limits, especially for Snodgrassella (Fig. S1). However, probability of growth at the lowest temperature tested $\left(12{ }^{\circ} \mathrm{C}\right)$ was positively associated with starting inoculum size (logistic regression, $p=0.0047$ ), which was not the case for growth at $44{ }^{\circ} \mathrm{C}$ (logistic regression, $p=0.74$ ). Hence, we regard the lower limit data as minimum estimates; many of these strains are likely to be able to grow at lower temperatures than indicated in Fig. S1, especially with larger starting inocula or a longer assay duration.

In addition to characterizing the heat-sensitivity of symbiont growth under a constant temperature, we also sought to determine the ability of bee gut symbionts to tolerate short-term exposure to more extreme heat. Exposure to temperatures above $35^{\circ} \mathrm{C}$ for $1 \mathrm{~h}$ clearly delayed subsequent growth in vitro (Fig. 2). Overall, however, Snodgrassella appears to be quite heattolerant in that all six strains assayed could recover from exposure to $48.7^{\circ} \mathrm{C}$. The exact limit was variable among strains. Although larger strain sample sizes are needed for a conclusive comparison, the three Apis-associated strains tended to be slightly more heat-tolerant than the three Bombus-associated strains (Fig. 2). Strain robustness to short-term exposures was not simply a function of the initial inoculum size. For example, the only two strains that could recover following exposure to $51.6^{\circ} \mathrm{C}$, wkB9 and wkB237, had the lowest inoculum sizes.

Even when thermal limits do not vary among symbiont strains, the thermal optima could vary. To address this, we conducted Snodgrassella growth assays in liquid media at $28^{\circ} \mathrm{C}$ and 35 ${ }^{\circ} \mathrm{C}$, representing temperatures closer to typical Bombus or Apis nest temperatures, respectively. Inoculum size (number of CFUs at the start of the growth assay) did not predict growth rate at 
207 either $28^{\circ} \mathrm{C}$ or $35^{\circ} \mathrm{C}$ (linear regression, $p=0.69$ and 0.82 , respectively). Interestingly,

208 bumblebee-associated Snodgrassella typically had higher growth rates than honeybee-associated

209 Snodgrassella (Fig. 3; two-way ANOVA, $p=0.0085$ ). We found no significant interaction

210 between host genus and temperature (two-way ANOVA, $p=0.24$ ), as would be expected if

211 symbiont growth were differentially adapted to the temperatures closest to those of their hosts'

212 nests. Instead, growth rates of most strains were higher at $35^{\circ} \mathrm{C}$ (Fig. 3; two-way ANOVA, $p=$

213 0.0059). Among bumblebee strains, those from host species inhabiting higher elevations were

214 not conspicuously better able to grow at the cooler temperature, $28^{\circ} \mathrm{C}$, than those from low-

215 elevation hosts (Fig. S2). In fact, the only strains that exhibited higher growth rates at $28{ }^{\circ} \mathrm{C}$ than

216 at $35{ }^{\circ} \mathrm{C}$ were derived from Apis mellifera (Fig. S2).

217 We next examined whether symbiont thermotolerance might influence fitness in vivo

218 using a colonization experiment with Snodgrassella alvi strain wkB12 and gnotobiotic

219 bumblebees. In vitro, this strain grows much more quickly at $35^{\circ} \mathrm{C}$ as compared with $28^{\circ} \mathrm{C}$ (Fig.

220 4B). No Snodgrassella colony-forming units (CFUs) were detected in any of the bees inoculated

221 with sterile buffer $\left(\mathrm{N}=15\right.$ reared at $29^{\circ} \mathrm{C}, \mathrm{N}=16$ reared at $\left.35^{\circ} \mathrm{C}\right)$. Among bees inoculated with

222 a standardized dose of $\sim 10^{6}$ Snodgrassella cells, gut colonization after 5 days was highly

223 dependent on the thermal environment (Fig. 4A). Accounting for the different source colonies

224 from which bees were obtained, CFU counts were significantly higher (by over 50-fold,

225 comparing medians) in the $35^{\circ} \mathrm{C}$ rearing treatment (linear mixed-effects model, $p<0.0001$ ).

226 Several bees reared at $29^{\circ} \mathrm{C}$ had no detectable Snodgrassella cells.

We also examined whether bee survival was influenced by the experimental treatments.

228 Overall, $92 \%$ of bees survived from adult emergence to dissection on day 5 . The probability of

229 survival was not affected by the inoculum (Snodgrassella vs. buffer alone) or the rearing 
230 temperature (logistic regression; inoculum $p=0.49$, temperature $p=0.33$ ) (Fig. S3). As only

231 bees that survived for the entire 5-day rearing period were used for CFU counts, we were not

232 able to examine whether gut colonization was predictive of survival.

\section{Discussion}

We first used in vitro assays to characterize bee symbiont thermal niches in a common

237 environment and without potential host-mediated effects. The experiments were based on a

238 collection of isolates of Snodgrassella and Gilliamella, which are ubiquitous, keystone members

239 of the social bee gut microbiome $[17,18]$. Overall, we found that these symbionts are quite heat-

240 tolerant relative to their hosts. All strains of both symbiont species can grow at a constant

241 temperature of $40^{\circ} \mathrm{C}$, and many can grow at $44{ }^{\circ} \mathrm{C}$ (Fig. 1). Likewise, another core symbiont,

242 Lactobacillus bombicola, has an optimal growth temperature around $40{ }^{\circ} \mathrm{C}$ [55]. In contrast,

243 honeybees and bumblebees do not normally allow their nests to reach temperatures above $35^{\circ} \mathrm{C}$,

244 which would harm brood development $[31,32,41-43,56]$.

245 Bees can, however, maintain higher body temperatures for brief periods while foraging.

246 For example, the abdomen (which contains the gut microbes [18]) of bumblebees foraging in full

247 sunlight may reach close to $40{ }^{\circ} \mathrm{C}$ [28]. The abdomen is also used to dissipate excess heat

248 generated in the thorax [57]. We used short-term heat treatments of Snodgrassella to mimic this

249 kind of temporary exposure, and found that bumblebee-associated strains could recover from an

250 hour-long exposure to at least $48.7^{\circ} \mathrm{C}$, while honeybee-associated strains could recover from at

251 least $50.4^{\circ} \mathrm{C}$ (Fig. 2). In contrast, measured lethal limits (broadly defined) of bumblebees and

252 honeybees are similar or lower, around $\sim 40-46{ }^{\circ} \mathrm{C}[58,59]$ and $\sim 50{ }^{\circ} \mathrm{C}[60-62]$, respectively. 
Bumblebees have experienced population declines partly linked to climate warming

254 [21,22]; in this study, we asked whether heat-sensitive symbionts could constitute one underlying

255 mechanism. The comparative robustness of Snodgrassella, Gilliamella, and Lactobacillus

256 bombicola to high temperatures in vitro suggests that the gut microbiome does not constrain bee

257 tolerance of heat. Rather, other factors rooted in host physiology, behavior, and ecology likely

258 explain the observed impacts of climate change on bumblebee populations [63-65]. However,

259 further experiments in vivo are needed to conclusively test whether the microbiome plays a role

260 in mediating effects of heat stress on bee populations.

Snodgrassella and Gilliamella are heat-tolerant not only compared to their hosts, but also

262 compared to many insect endosymbionts. For example, aphids, weevils, carpenter ants, and

263 stinkbugs all have obligate associations with highly heat-sensitive endosymbionts

$264[10,13,14,24,66]$. One trait these symbionts share is strict maternal inheritance; this transmission

265 mode enforces a clonal population structure that results in genome degeneration and, ultimately,

266 impaired heat tolerance [7-9]. In contrast, social transmission of gut symbionts permits the strain

267 mixing and recombination that is more typical for free-living bacteria. While mostly vertically

268 transmitted between colonies, bee gut symbionts likely maintain larger population sizes and

269 undergo recombination more frequently than endosymbionts. All bee gut symbiont species are

270 culturable outside the host [67] and possess genomes that do not exhibit the hallmarks of

271 degenerative evolution [40].

272 Like many gut microbes, bee gut symbionts experience a brief but potentially important

273 ex vivo phase during transmission. Selection for persistence on nest substrates or in the

274 environment (e.g., flowers) may influence their heat tolerance. In vivo selective pressures related

275 to the unique thermoregulatory behavior of social bees may further explain the broad thermal 
range of bee symbionts. A bee-inhabiting microbial population will experience a wide range of body temperatures as its hosts forage in the environment, thermoregulate nests, and overwinter.

278 In the future, comparisons to non-bee-associated bacterial relatives (e.g., [68]) would be useful to

279 reconstruct how evolution in social bees specifically has shaped the thermal niches of the bee gut

280 microbiome.

While generally heat-tolerant relative to their hosts and to other bacterial symbionts of insects, Snodgrassella and Gilliamella strains do vary in thermal traits. This variability manifests

283 as higher heat tolerance for honeybee versus bumblebee strains (Fig. 1, Fig. 2), a pattern that

284 roughly matches the corresponding thermal traits of their hosts. For example, honeybees

287 diapause in winter [26,62,69]. Our findings are consistent with previously observed correlations

288 between symbiont thermotolerance and the local thermal environment [24,70-72]. In the case of 289 bees, even if divergent thermal niches of symbionts do not affect hosts, they could affect the 290 potential for strains to successfully disperse between host colonies or even species, ultimately 291 influencing their biogeography and degree of host specialization.

293 performance (i.e., relative growth rate at two temperatures). Bumblebee strains uniformly grew

294 faster at $35^{\circ} \mathrm{C}$, while most Apis mellifera strains grew slightly faster at $28^{\circ} \mathrm{C}$ (Fig. 3, Fig. S2).

295 This pattern is the opposite of what would be expected if the ambient temperature in active 296 colonies primarily determines the optimal growth temperature of symbionts, because honeybees

297 generally maintain warmer nests. We speculate that overwintering biology may explain the $A$.

298 mellifera-derived strains' comparatively higher growth rates at the cool assay temperature. 
Unlike bumblebees, in winter, $A$. mellifera workers form active clusters that maintain aboveambient, but cool temperatures (average $\sim 21^{\circ} \mathrm{C}$; [32]).

302 Lactobacillus bombicola [55], grow faster at a temperature exceeding that of their nests (Fig. 3)?

303 We suggest that the answer lies in the bee abdomen - the microenvironment inhabited by the

304 symbionts, and a structure whose temperature is affected by host thermoregulatory behavior.

305 Specifically, both workers and queens (and occasionally males [73]) of bumblebees incubate

306 larvae and pupae by placing their abdomen directly onto brood structures and elevating its temperature to $\sim 35^{\circ} \mathrm{C}$ or above, exceeding ambient temperatures in the nest [74,75]. Given that bumblebees spend much of their time performing this behavior $[41,74,76]$ — especially foundress

309 queens, the sole source of microbes for the colony — symbiont growth may be adapted to the 310 locally heated conditions within the abdomen.

To further explore this possibility, we tested whether the warm-shifted growth preference

312 of bumblebee-associated Snodgrassella (Fig. 3) might have fitness effects in vivo. We conducted 313 an experiment on gnotobiotic Bombus impatiens, using conditions (microcolonies lacking brood)

314 in which incubation behavior is limited, and thus abdominal temperatures are expected to more 315 closely match the chosen ambient rearing temperatures $\left(29^{\circ} \mathrm{C}\right.$ and $\left.35^{\circ} \mathrm{C}\right)$. Previous literature 316 hinted that these temperatures might affect Snodgrassella colonization of $B$. impatiens. In one 317 study, experimental inoculation of Bombus impatiens with Snodgrassella resulted in 100\%

318 colonization and consistently high titers [40], whereas a later study of B. impatiens reported 319 erratic colonization and frequently low titers [51]. These studies differed in the temperature at 320 which bees were reared, with the high colonization rate observed at $34{ }^{\circ} \mathrm{C}$ and erratic 321 colonization at $\sim 26^{\circ} \mathrm{C}$. In line with these previous studies, when we directly examined the effect 
322 of the thermal environment on Snodgrassella colonization, we found that colonization was

323 variable and occasionally unsuccessful in bees reared at $29^{\circ} \mathrm{C}$ (Fig. 4A).

One possible explanation for this result has to do with the fact that the focal strain used,

325 Snodgrassella alvi wkB12, was not isolated from B. impatiens but rather B. bimaculatus. If

326 bumblebees control colonization by other species' or even colonies' symbiont strains, as has

327 been suggested [51], robust colonization at $35^{\circ} \mathrm{C}$ may actually reflect a weakening of strain-

328 specific filtering mechanisms at this unnaturally high rearing temperature. However, we

329 currently lack evidence for such mechanisms.

330 Another explanation for this result is based in the thermal niche of bumblebee-associated

331 Snodgrassella strains. Snodgrassella alvi wkB12, like the others tested, grows more quickly at

$33235^{\circ} \mathrm{C}$ than $28^{\circ} \mathrm{C}$ in vitro (Fig. 4B). In bees reared in microcolonies at $29^{\circ} \mathrm{C}$-which we expect

333 to have similar abdominal temperatures because of restricted thermoregulatory behavior-

334 ingested Snodgrassella cells may simply be unable to replicate quickly enough to establish a

335 stable population before being lost to defecation. However, once established, they appear to be

336 quite robust to a wide range of temperatures [37]. A subset of bees reared at $29^{\circ} \mathrm{C}$ did acquire

337 high Snodgrassella titers that exceeded the number of cells in the inoculum, implying replication

338 in the gut (Fig. 4A). One possibility is that these individuals had begun to incubate the provided

339 pollen lump, a behavior which has been observed in microcolonies (e.g., [77]). As a

340 consequence, they may have maintained higher abdominal temperatures conducive to

341 Snodgrassella colonization. Analogously, in a normal bumblebee nest, individual-level

342 thermoregulatory behaviors may be important in enabling establishment of the gut microbiome.

343 We note that our in vitro and in vivo experiments were conducted on single isolates, which

344 enables controlled assays of strain-level thermotolerance but may miss important community 
345 interactions that occur within the bee gut. Microbial thermotolerance can be modified by co-

346 occurring microbes or viruses (e.g., [78-81]), and phage have recently been documented from

347 the bee gut microbiome [82,83]. Community interactions may also help explain why

348 monocolonizations of Snodgrassella into bees reared at $29^{\circ} \mathrm{C}$ or below have been erratic (Fig.

$3494 \mathrm{~A}$ and [51]), while fecal transplants at these temperatures have not $[36,50]$. Another difference

350 between Snodgrassella colonization from cultured isolates and from fecal transplants is in the

351 quantity and physiological state of the cells that bees ingest. These factors could also help

352 symbionts overcome a temperature barrier to gut colonization.

\section{Conclusions}

We have argued that the gut microbiome is probably not a major constraint on how social

357 bees respond to heat stress. However, as their growth is somewhat cold-sensitive, symbionts

358 could be affected by other kinds of environmental stressors that disrupt host thermoregulatory

359 behavior. For example, neonicotinoid pesticides interfere with $B$. impatiens thermoregulation

360 [84]. Pesticides could thus indirectly affect colonization by Snodgrassella and perhaps other key

361 symbionts, a process we have shown to be temperature-sensitive. Whether symbionts indeed

362 constrain (or even improve [85]) bee responses to heat and other stressors remains an important

363 priority for future research, as bees and the pollination services they provide continue to face

364 serious challenges.

365 Our findings are also relevant beyond bees. We have identified a potential feedback

366 between host behavior and the microbiome, a topic that has recently garnered substantial interest

367 [86,87]. Specifically, we suggest that social bee thermoregulatory behaviors have provided 
368 elevated and buffered microenvironments (nests and individual bodies), shaping the evolution of

369 their symbionts' thermal niches. Additionally, there are parallels between bees and mammals,

370 which also create warm gut environments and harbor socially transmitted gut symbionts. Bees

371 could be a useful model to understand how these factors influence symbiont thermotolerance, a

372 little-studied trait in humans and other mammals.

373

374 Data Availability

Primary data and $\mathrm{R}$ code used for analyses and visualizations are publicly available at the

377 figshare repository: https://doi.org/10.6084/m9.figshare.12486395.

379 Acknowledgments

This research was supported by a postdoctoral fellowship (2018-08156) from the USDA

382 National Institute of Food and Agriculture to TJH, a University of Texas Undergraduate

383 Research Fellowship to EL, and National Institutes of Health award (R35GM131738) to NAM.

384 We also thank S. Leonard and E. Powell for helpful advice and K. Hammond for assistance. 


\section{References}

389 1. Cahill AE et al. 2013 How does climate change cause extinction? Proc. R. Soc. B 280, 20121890. (doi:10.1098/rspb.2012.1890)

2. Urban MC. 2015 Accelerating extinction risk from climate change. Science 348, 571-573.

392 (doi:10.1126/science.aaa4984)

3. Harrington R, Woiwod I, Sparks T. 1999 Climate change and trophic interactions. Trends in Ecology \& Evolution 14, 146-150. (doi:10.1016/S0169-5347(99)01604-3)

4. Colwell RK, Dunn RR, Harris NC. 2012 Coextinction and Persistence of Dependent Species in a Changing World. Annual Review of Ecology, Evolution, and Systematics 43, 183-203. (doi:10.1146/annurev-ecolsys-110411-160304)

5. Kiers TE, Palmer TM, Ives AR, Bruno JF, Bronstein JL. 2010 Mutualisms in a changing world: an evolutionary perspective. Ecology Letters 13, 1459-1474. (doi:10.1111/j.14610248.2010.01538.x)

6. Wernegreen JJ. 2012 Mutualism meltdown in insects: bacteria constrain thermal adaptation. 
8. Fares MA, Ruiz-González MX, Moya A, Elena SF, Barrio E. 2002 GroEL buffers against deleterious mutations. Nature 417, 398-398. (doi:10.1038/417398a)

9. Moran NA, McCutcheon JP, Nakabachi A. 2008 Genomics and Evolution of Heritable Bacterial Symbionts. Annual Review of Genetics 42, 165-190. (doi:10.1146/annurev.genet.41.110306.130119)

10. Corbin C, Heyworth ER, Ferrari J, Hurst GDD. 2017 Heritable symbionts in a world of varying temperature. Heredity 118, 10-20. (doi:10.1038/hdy.2016.71)

413 11. Moran NA. 2016 When obligate partners melt down. mBio 7, 7-9.

$414 \quad$ (doi:10.1128/mBio.01904-16)

415 12. Dunbar HE, Wilson ACC, Ferguson NR, Moran NA. 2007 Aphid thermal tolerance is 416 governed by a point mutation in bacterial symbionts. PLoS Biology 5, e96.

417 (doi:10.1371/journal.pbio.0050096)

418 13. Fan Y, Wernegreen JJ. 2013 Can’t Take the Heat: High Temperature Depletes Bacterial 419 Endosymbionts of Ants. Microb Ecol 66, 727-733. (doi:10.1007/s00248-013-0264-6)

420 14. Kikuchi Y, Tada A, Musolin DL, Hari N, Hosokawa T, Fujisaki K. 2016 Collapse of Insect

421 Gut Symbiosis under Simulated Climate Change. 7, 1-8. (doi:10.1128/mBio.01578-

$422 \quad$ 16.Editor)

423 15. Weis VM. 2008 Cellular mechanisms of Cnidarian bleaching: stress causes the collapse of 424 symbiosis. Journal of Experimental Biology 211, 3059-3066. (doi:10.1242/jeb.009597) 
425 16. Webster NS, Cobb RE, Negri AP. 2008 Temperature thresholds for bacterial symbiosis with a sponge. ISME J 2, 830-842. (doi:10.1038/ismej.2008.42)

427 17. Kwong W et al. 2017 Dynamic microbiome evolution in social bees. Science Advances 3 , $428 \quad$ e1600513. (doi:10.1126/sciadv.1600513)

429 18. Kwong WK, Moran NA. 2016 Gut microbial communities of social bees. Nature Reviews Microbiology 14, 374-384. (doi:10.1038/nrmicro.2016.43)

19. Cameron SA, Sadd BM. 2020 Global Trends in Bumble Bee Health. Annu. Rev. Entomol. 65, 209-232. (doi:10.1146/annurev-ento-011118-111847)

20. Potts SG. 2015 Global pollinator declines: Trends, impacts and drivers. Trends in Ecology and Evolution 10, 345-353. (doi:10.1016/j.tree.2010.01.007)

21. Kerr JT et al. 2015 Climate change impacts on bumblebees converge across continents. Science 349, 177-180. (doi:10.1126/science.aaa7031) among bumble bees across continents. Science 367, 685-688. (doi:10.1126/science.aax8591)

23. Powell E, Ratnayeke N, Moran NA. 2016 Strain diversity and host specificity in a specialized gut symbiont of honeybees and bumblebees. Molecular Ecology 25, 4461-4471. thermal tolerance of insect host species. Proceedings of the National Academy of Sciences 116, 24712-24718. (doi:10.1073/pnas.1915307116) 
445 25. Heinrich B, Esch H. 1994 Thermoregulation in bees. American Scientist 82, 164-170.

446 26. Southwick EE, Heldmaier G. 1987 Temperature Control in Honey Bee Colonies. BioScience

447 37, 395-399. (doi:10.2307/1310562)

448 27. Heinrich B. 1979 Bumblebee Economics. Cambridge, Massachusetts: Harvard University

$449 \quad$ Press.

450

451

452

453

454

455

28. Heinrich B. 1972 Energetics of temperature regulation and foraging in a bumblebee, Bombus terricola Kirby. Journal of Comparative Physiology 77, 49-64. (doi:10.1007/BF00696519)

29. Heinrich B. 1972 Patterns of endothermy in bumblebee queens, drones and workers. $J$. Comp. Physiol. 77, 65-79. (doi:10.1007/BF00696520)

30. Heinrich B. 1974 Thermoregulation in bumblebees: I. Brood incubation by Bombus vosnesenskii queens. J. Comp. Physiol. 88, 129-140. (doi:10.1007/BF00695404)

31. Tan K, Yang S, Wang Z-W, Radloff SE, Oldroyd BP. 2012 Differences in foraging and broodnest temperature in the honey bees Apis cerana and A. mellifera. Apidologie 43, 618623. (doi:10.1007/s13592-012-0136-y)

32. Fahrenholz L, Lamprecht I, Schricker B. 1989 Thermal investigations of a honey bee colony: thermoregulation of the hive during summer and winter and heat production of members of different bee castes. J Comp Physiol B 159, 551-560. (doi:10.1007/BF00694379)

33. Kešnerová L, Emery O, Troilo M, Liberti J, Erkosar B, Engel P. 2020 Gut microbiota structure differs between honeybees in winter and summer. ISME J 14, 801-814. (doi:10.1038/s41396-019-0568-8) 
34. Koch H, Abrol DP, Li J, Schmid-Hempel P. 2013 Diversity and evolutionary patterns of bacterial gut associates of corbiculate bees. Molecular Ecology 22, 2028-2044. (doi:10.1111/mec.12209)

35. Powell JE, Martinson VG, Urban-Mead K, Moran NA. 2014 Routes of Acquisition of the Gut Microbiota of the Honey Bee Apis mellifera. Applied and Environmental Microbiology

36. Koch H, Schmid-Hempel P. 2011 Socially transmitted gut microbiota protect bumble bees 80, 7378-7387. (doi:10.1128/AEM.01861-14) Temperature dependence of parasitic infection and gut bacterial communities in bumble

38. Martinson VG, Moy J, Moran NA. 2012 Establishment of characteristic gut bacteria during development of the honeybee worker. Applied and Environmental Microbiology 78, 2830-

39. Michener CD. 1979 Biogeography of the Bees. Annals of the Missouri Botanical Garden 66, 277. (doi:10.2307/2398833) 
41. Vogt FD. 1986 Thermoregulation in Bumblebee Colonies. I. Thermoregulatory versus Brood-Maintenance Behaviors during Acute Changes in Ambient Temperature. Physiological Zoology 59, 55-59.

42. Gardner KE, Foster RL, O’Donnell S. 2007 Experimental analysis of worker division of labor in bumblebee nest thermoregulation (Bombus huntii, Hymenoptera: Apidae). Behav Ecol Sociobiol 61, 783-792. (doi:10.1007/s00265-006-0309-7)

43. Weidenmüller A, Kleineidam C, Tautz J. 2002 Collective control of nest climate parameters in bumblebee colonies. Animal Behaviour 63, 1065-1071. (doi:10.1006/anbe.2002.3020)

44. Jones JC, Myerscough MR, Graham S, Oldroyd BP. 2004 Honey bee nest thermoregulation: Diversity promotes stability. Science 305, 402-404. (doi:10.1126/science.1096340)

45. Steele MI, Kwong WK, Whiteley M, Moran NA. 2017 Diversification of Type VI Secretion System Toxins Reveals Ancient Antagonism among Bee Gut Microbes. mBio 8, e01630-17. Metabolism of toxic sugars by strains of the bee gut symbiont Gilliamella apicola. mBio 7,

47. Williams P, Thorp R, Richardson L, Colla S. 2014 Bumble Bees of North America. e01326-16. (doi:10.1128/mBio.01326-16)

48. R Core Team. 2016 R: A language and environment for statistical computing. Vienna, 
49. Sprouffske K, Wagner A. 2016 Growthcurver: an R package for obtaining interpretable metrics from microbial growth curves. BMC Bioinformatics 17, 172. (doi:10.1186/s12859016-1016-7)

50. Näpflin K, Schmid-Hempel P. 2018 Host effects on microbiota community assembly. Journal of Animal Ecology 87, 331-340. (doi:10.1111/1365-2656.12768)

51. Sauers LA, Sadd BM. 2019 An interaction between host and microbe genotypes determines colonization success of a key bumble bee gut microbiota member. Evolution 73, 2333-2342. (doi:10.1111/evo.13853)

52. Klinger EG, Camp AA, Strange JP, Cox-Foster D, Lehmann DM. 2019 Bombus (Hymenoptera: Apidae) Microcolonies as a Tool for Biological Understanding and Pesticide Risk Assessment. Environmental Entomology 48, 1249-1259.

53. Leza M, Watrous KM, Bratu J, Woodard SH. 2018 Effects of neonicotinoid insecticide exposure and monofloral diet on nest-founding bumblebee queens. Proceedings of the Royal Society B: Biological Sciences 285, 20180761. (doi:10.1098/rspb.2018.0761) Effects Models. See https://CRAN.R-project.org/package=nlme. of a bumblebee parasite by an intestinal symbiont. Proceedings of the Royal Society B $\mathbf{2 8 5}$, 20182041. (doi:http://dx.doi.org/10.1098/rspb.2018.2041) 
524 56. Jones JC. 2004 Honey Bee Nest Thermoregulation: Diversity Promotes Stability. Science 305, 402-404. (doi:10.1126/science.1096340)

57. Heinrich B. 1976 Heat exchange in relation to blood flow between thorax and abdomen in bumblebees. The Journal of experimental biology 64, 561-85.

58. Hamblin AL, Youngsteadt E, López-Uribe MM, Frank SD. 2017 Physiological thermal limits predict differential responses of bees to urban heat-island effects. Biology Letters 13,

59. Oyen KJ, Giri S, Dillon ME. 2016 Altitudinal variation in bumble bee (Bombus) critical 20170125. (doi:10.1098/rsbl.2017.0125)

60. Kovac H, Käfer H, Stabentheiner A, Costa C. 2014 Metabolism and upper thermal limits of Apis mellifera carnica and A. m. ligustica. Apidologie 45, 664-677. (doi:10.1007/s13592-

61. Käfer H, Kovac H, Stabentheiner A. 2012 Upper thermal limits of honeybee (Apis mellifera) and yellowjacket (Vespula vulgaris) foragers. Mitt. Dtsch. Ges. Allg. Angew. Ent. 18, 267-

62. Ken T, Hepburn HR, Radloff SE, Yusheng Y, Yiqiu L, Danyin Z, Neumann P. 2005 Heatballing wasps by honeybees. Naturwissenschaften 92, 492-495. (doi:10.1007/s00114-005- 
64. Rasmont P, Iserbyt S. 2012 The Bumblebees Scarcity Syndrome: Are heat waves leading to local extinctions of bumblebees (Hymenoptera: Apidae: Bombus)? Annales de la Société entomologique de France (N.S.) 48, 275-280. (doi:10.1080/00379271.2012.10697776)

65. Woodard SH. 2017 Bumble bee ecophysiology: integrating the changing environment and the organism. Current Opinion in Insect Science 22, 101-108.

66. Anbutsu $\mathrm{H}$ et al. 2017 Small genome symbiont underlies cuticle hardness in beetles.

$$
\text { (doi:10.1016/j.cois.2017.06.001) }
$$

67. Engel P, James RR, Koga R, Kwong WK, McFrederick QS, Moran NA. 2013 Standard methods for research on Apis mellifera gut symbionts. Journal of Apicultural Research 52, 1-24. (doi:10.3896/IBRA.1.52.4.07)

68. Wertz JT, Breznak JA. 2007 Stenoxybacter acetivorans gen. nov., sp. nov., an AcetateOxidizing Obligate Microaerophile among Diverse O2-Consuming Bacteria from Termite

69. Benjamin P. Oldroyd, Siriwat Wongsiri. 2006 Asian Honey Bees: Biology, Conservation, and Human Interactions. Cambridge, Massachusetts: Harvard University Press. adaptation constrains the distribution potential of heat-tolerant Symbiodinium from the 
563 71. Mueller UG et al. 2011 Evolution of cold-tolerant fungal symbionts permits winter

564 fungiculture by leafcutter ants at the northern frontier of a tropical ant-fungus symbiosis.

565 Proceedings of the National Academy of Sciences 108, 4053-4056.

$566 \quad$ (doi:10.1073/pnas.1015806108)

567 72. Okada S, Gordon DM. 2001 Host and geographical factors influence the thermal niche of 568 enteric bacteria isolated from native Australian mammals. Molecular Ecology 10, 24992513. (doi:10.1046/j.0962-1083.2001.01384.x)

73. Cameron SA. 1985 Brood care by male bumble bees. Proceedings of the National Academy of Sciences 82, 6371-6373. (doi:10.1073/pnas.82.19.6371) vosnesenskii. Nature 239, 223-225.

574 75. Kelemen E, Dornhaus A. 2018 Lower temperatures decrease worker size variation but do not affect fine-grained thermoregulation in bumble bees. Behav Ecol Sociobiol 72, 170. (doi:10.1007/s00265-018-2577-4) Bumble Bees, Bombus bifarius nearcticus (Hymenoptera: Apidae). Ethology 107, 387-399. (doi:10.1046/j.1439-0310.2001.00668.x) microcolonies of bumble bees (Bombus terrestris L.). Ecol Entomol 44, 601-609. 
583 78. Burke G, Fiehn O, Moran N. 2010 Effects of facultative symbionts and heat stress on the metabolome of pea aphids. ISME Journal 4, 242-252. (doi:10.1038/ismej.2009.114)

79. Ma L, Calfee BC, Morris JJ, Johnson ZI, Zinser ER. 2018 Degradation of hydrogen peroxide at the ocean's surface: the influence of the microbial community on the realized thermal niche of Prochlorococcus. ISME J 12, 473-484. (doi:10.1038/ismej.2017.182)

80. Bordenstein SR, Bordenstein SR. 2011 Temperature Affects the Tripartite Interactions between Bacteriophage WO, Wolbachia, and Cytoplasmic Incompatibility. PLoS ONE 6, e29106. (doi:10.1371/journal.pone.0029106)

81. Marquez LM, Redman RS, Rodriguez RJ, Roossinck MJ. 2007 A Virus in a Fungus in a Plant: Three-Way Symbiosis Required for Thermal Tolerance. Science 315, 513-515. (doi:10.1126/science.1136237)

82. Deboutte W, Beller L, Yinda CK, Maes P, de Graaf DC, Matthijnssens J. 2020 Honey-beeassociated prokaryotic viral communities reveal wide viral diversity and a profound metabolic coding potential. Proc Natl Acad Sci USA 117, 10511-10519. (doi:10.1073/pnas.1921859117)

83. Bonilla-Rosso G, Steiner T, Wichmann F, Bexkens E, Engel P. 2020 Honey bees harbor a diverse gut virome engaging in nested strain-level interactions with the microbiota. Proc Natl Acad Sci USA 117, 7355-7362. (doi:10.1073/pnas.2000228117) 
603 85. Lemoine MM, Engl T, Kaltenpoth M. 2020 Microbial symbionts expanding or constraining

604 abiotic niche space in insects. Current Opinion in Insect Science 39, 14-20.

$605 \quad$ (doi:10.1016/j.cois.2020.01.003)

606 86. Lewis Z, Lizé A. 2015 Insect behaviour and the microbiome. Current Opinion in Insect

607 Science 9, 86-90. (doi:10.1016/j.cois.2015.03.003)

608 87. Sherwin E, Bordenstein SR, Quinn JL, Dinan TG, Cryan JF. 2019 Microbiota and the social

609 brain. Science 366, eaar2016. (doi:10.1126/science.aar2016) 


\section{$611 \quad$ Figures}

612
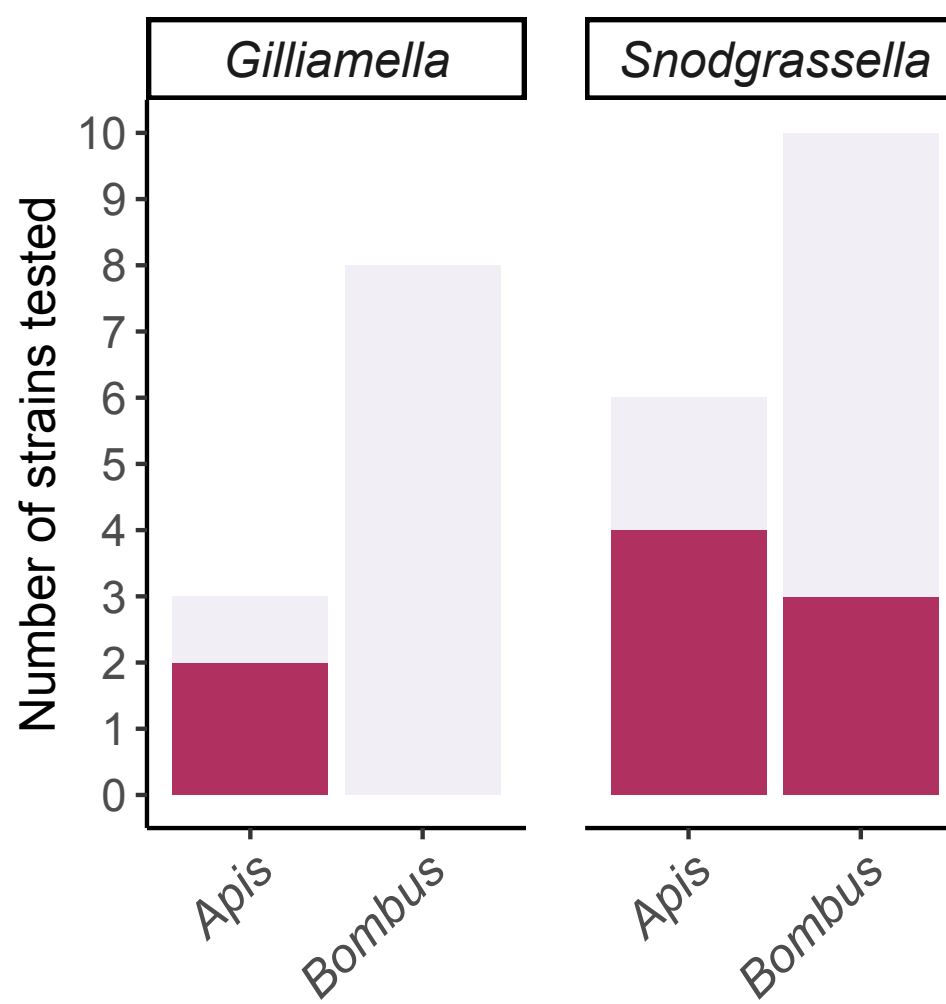

613

614 Figure 1. Ability of strains of two core bee gut symbionts, Gilliamella and Snodgrassella, to

615 grow after $48 \mathrm{~h}$ incubation on solid media at $44^{\circ} \mathrm{C}$. Honeybee (Apis) strains tend to be better

616 able to grow at this temperature than bumblebee (Bombus) strains. Thermal limits broken down

617 by host species of origin are shown in Fig. S1. 

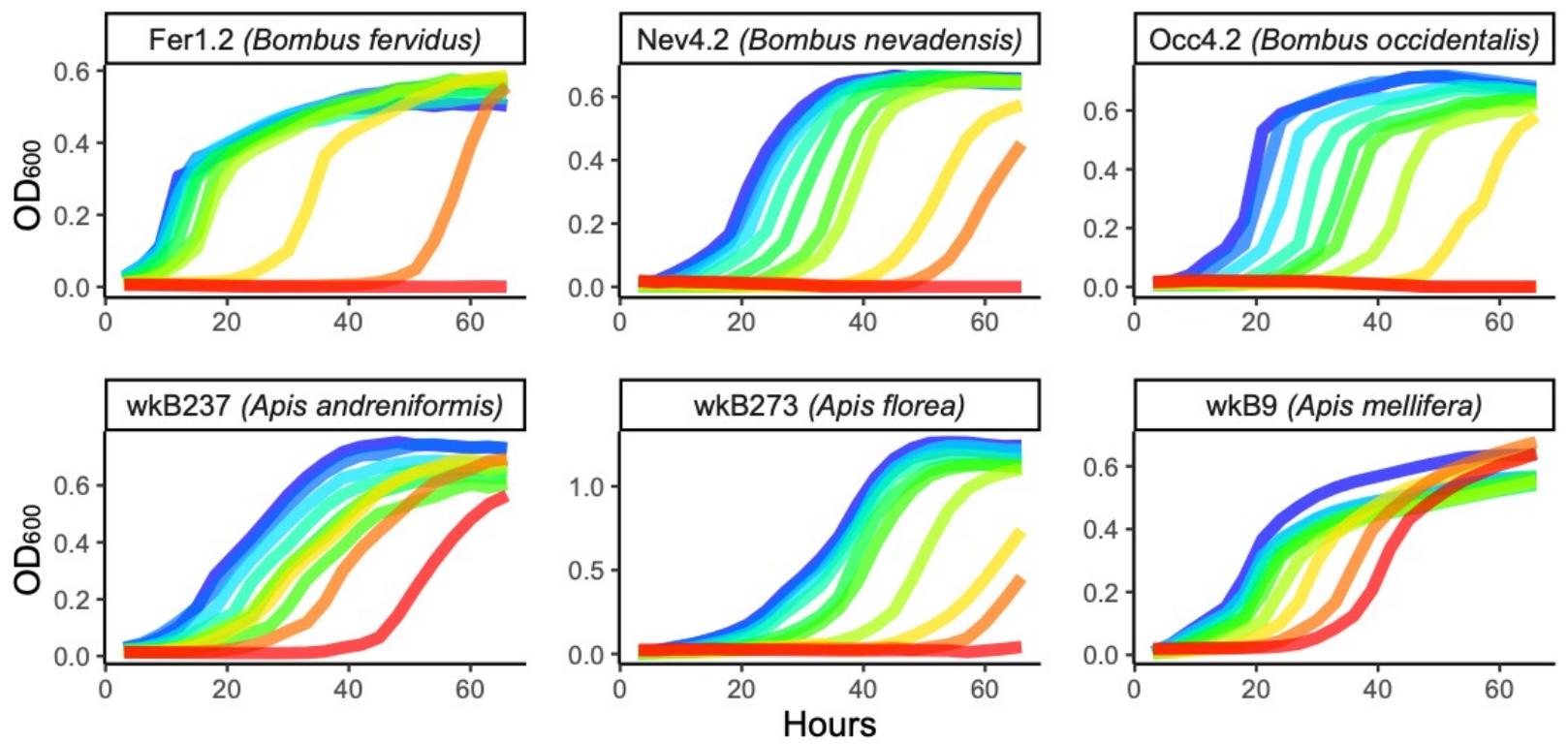

621 Figure 2. In vitro growth of the core bee gut symbiont Snodgrassella following short-term

622 exposure to high temperatures. Curves represent growth at $35^{\circ} \mathrm{C}$ and $5 \% \mathrm{CO}_{2}$ following a $1 \mathrm{~h}$

623 heat stress treatment applied using a gradient thermocycler, showing the mean $\mathrm{OD}_{600}$ values of

624 three replicates per strain per temperature. The host species from which each strain was isolated

625 is indicated. Snodgrassella is generally robust to high temperatures, though tolerance varied

626 among strains. Only two Apis-associated isolates were able to recover from $51.6^{\circ} \mathrm{C}$, the

627 maximum temperature tested.

628

629 


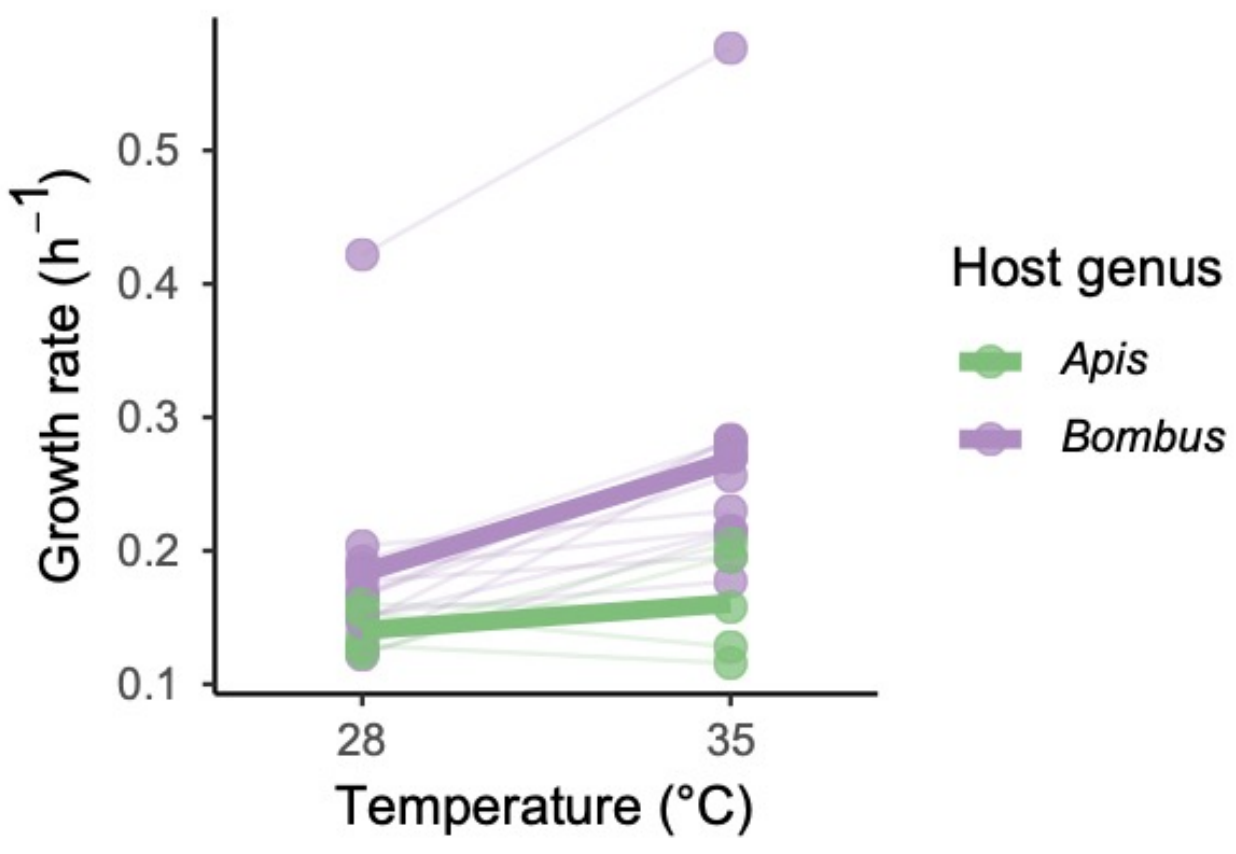

632 Figure 3. In vitro growth rates of Snodgrassella strains at $28^{\circ} \mathrm{C}$ and $35^{\circ} \mathrm{C}$. Dots connected by

633 thin lines represent the maximum growth rates of each strain, colored by whether the strains were

634 isolated from honeybees (Apis) or bumblebees (Bombus). Thick lines connect the median growth

635 rate for Apis or Bombus strains at each incubation temperature. All bumblebee Snodgrassella

636 grow faster at $35^{\circ} \mathrm{C}$, a temperature significantly higher than typical bumblebee nests. $\mathrm{N}=5$ Apis

637 strains, $\mathrm{N}=15$ Bombus strains. 
A)

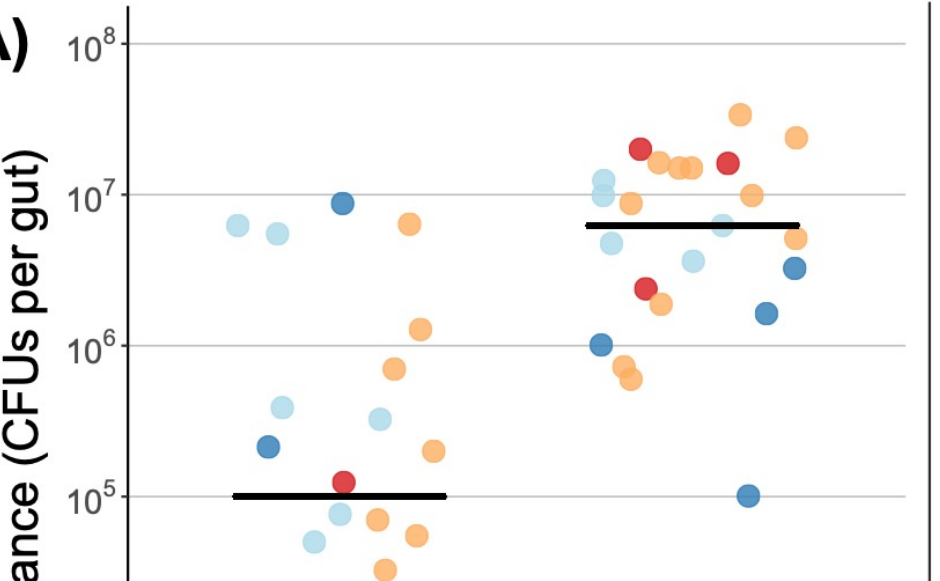

Figure 4. A) Effects of the thermal environment on colonization of Snodgrassella alvi wkB12 in

\section{Snodgrassella titer for each temperature treatment. Bees fed a sterile buffer and maintained}

645 under the same conditions had no detectable Snodgrassella colonization (not shown). CFUs = colony-forming units; ND = not detected. B) Growth of Snodgrassella alvi wkB12 in vitro when

647 incubated at $28^{\circ} \mathrm{C}$ versus $35^{\circ} \mathrm{C}$. Growth curves represent the mean $\mathrm{OD}_{600}$ values of three replicates per temperature. 


\section{Supplemental Figures}

652

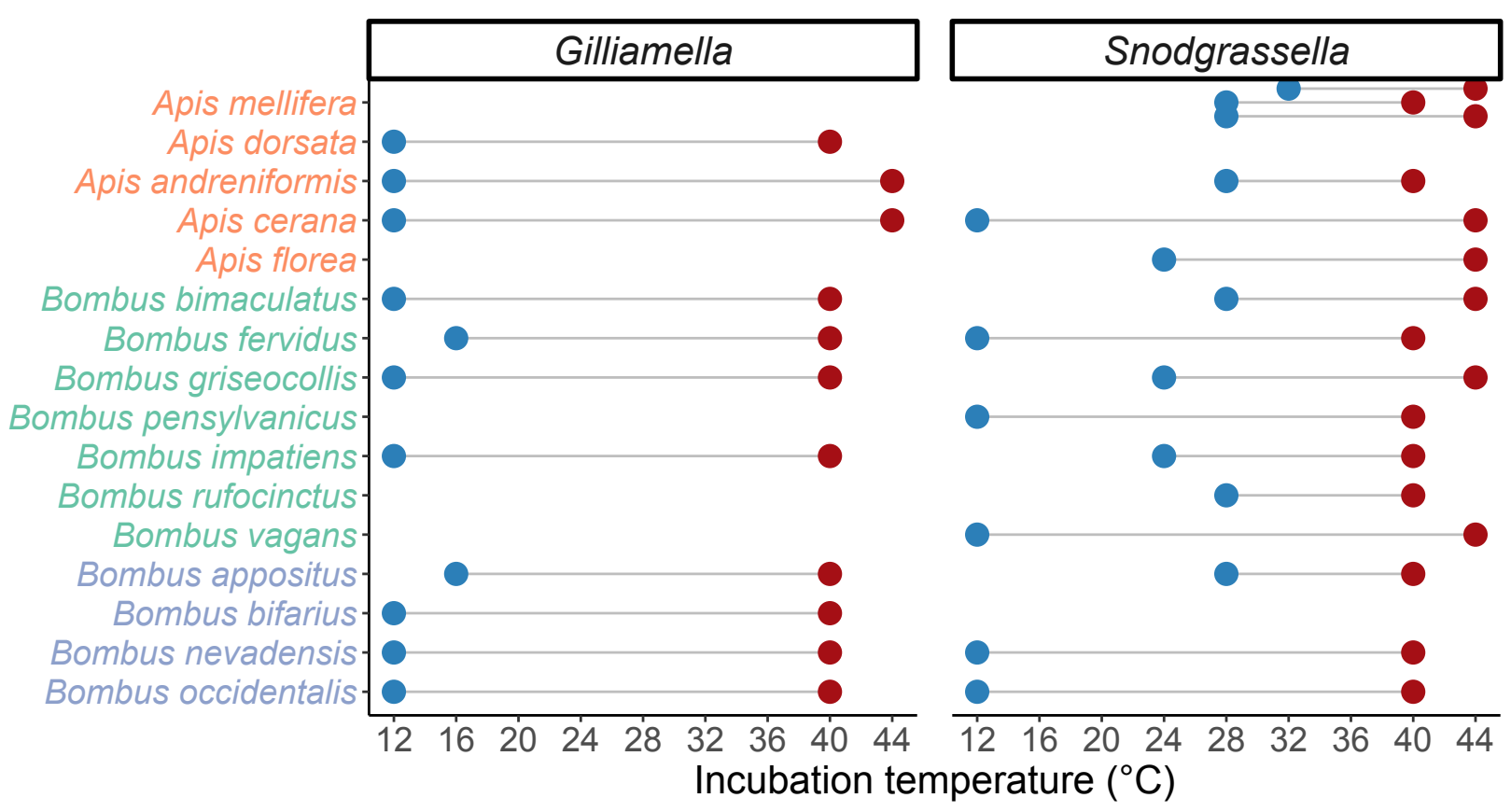

654 Figure S1. Thermal limits to growth in vitro for two bee gut symbiont species. Dots represent the

655 minimum and maximum temperatures at which a given strain exhibited growth after $48 \mathrm{~h}$ of

656 incubation on solid media. No growth was evident for any strain at $48^{\circ} \mathrm{C}$, and temperatures

657 below $12{ }^{\circ} \mathrm{C}$ were not tested. Aside from Apis mellifera, from which three Snodgrassella isolates

658 were assayed, one symbiont isolate per host species was assayed; note that blank spaces indicate

659 untested host/symbiont combinations. The four bumble bee host species that typically inhabit

660 high-elevation habitats are labeled in blue at the bottom of the plot. Lower limits to growth

661 should be considered as minimum estimates of cold tolerance, given the sensitivity of this metric

662 to the starting inoculum size (see Results). 


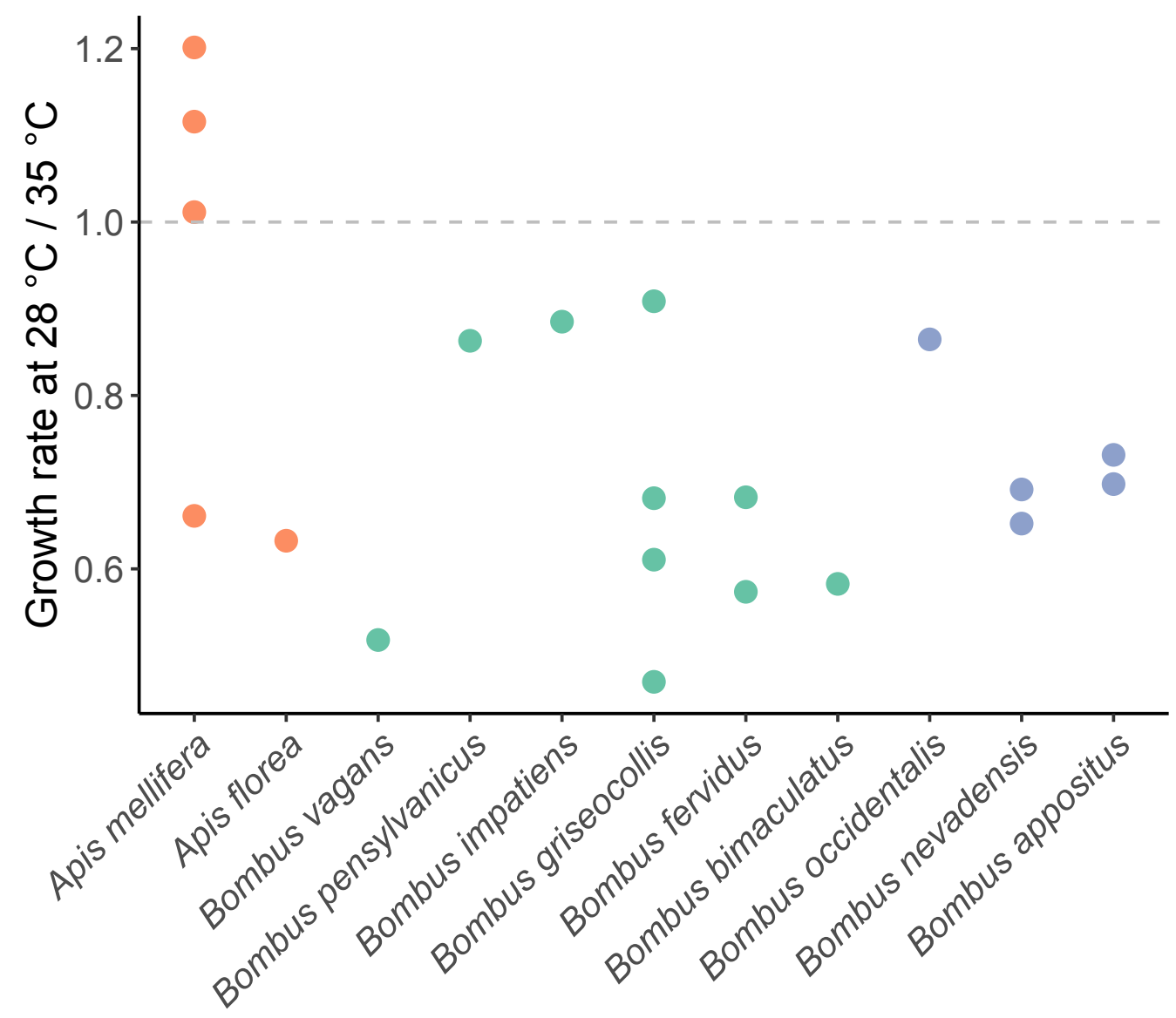

665 Figure S2. Growth rates of Snodgrassella at $28{ }^{\circ} \mathrm{C}$ versus $35^{\circ} \mathrm{C}$ in vitro. The dashed line

666 indicates equivalent growth rates between the two temperatures. Dots represent individual

667 Snodgrassella strains, and strains from bumble bee species that typically inhabit high-elevation

668 habitats are labeled in blue. The one $A$. mellifera-derived strain that grew faster at $35{ }^{\circ} \mathrm{C}$ is

669 wkB2, which has been frequently cultured in the laboratory at $35^{\circ} \mathrm{C}$ (see Methods). Its thermal

670 performance may therefore reflect laboratory evolution. 


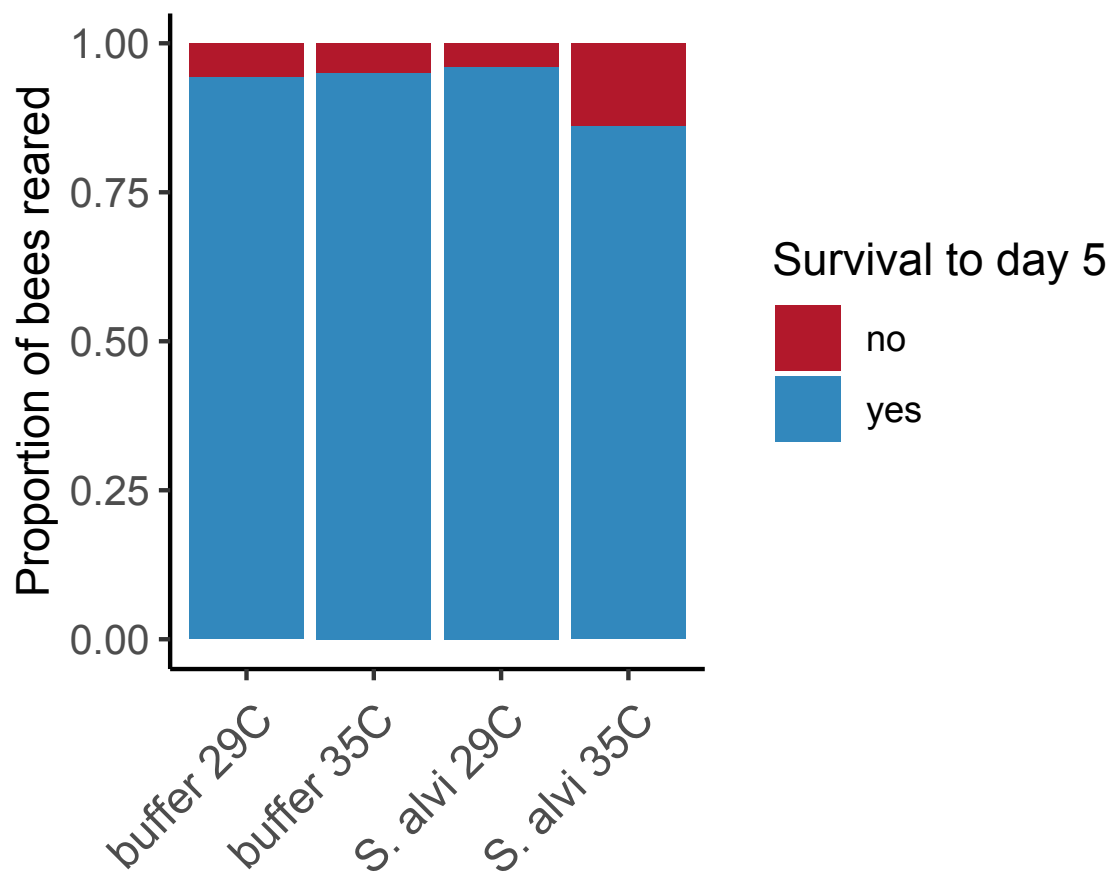

673 Figure S3. Survival outcomes of gnotobiotic bumble bees from adult emergence through five

674 days of rearing. Number of bees inoculated with buffer alone: 18 at $29{ }^{\circ} \mathrm{C}, 20$ at $35^{\circ} \mathrm{C}$. Number

675 of bees inoculated with Snodgrassella alvi wkB12: 25 at $29{ }^{\circ} \mathrm{C}, 29$ at $35^{\circ} \mathrm{C}$. 